\section{BRAIN}

\section{Strange bedfellows}

Ghrelin and dopamine receptors interact in the brain to form heterodimers with unexpected functional properties, according to Andras Kern et al. (Neuron 73, 317-332).

Receptors for ghrelin, an appetite-inducing gut hormone, exist in the brain, even in areas inaccessible to the hormone, raising questions about the functional relevance of these receptors. Kern et al. carried out a series of structurefunction, mutational and biochemical in vitro studies to discover an allosteric interaction between ghrelin and dopamine D2 receptors that alters the functional responses of the monomeric ghrelin or dopamine D2 receptors.

To establish the role of this interaction in vivo, the authors used a mouse model of anorexia induced by cabergoline, an agonist of the dopamine receptor D2 subtype used to treat Parkinson's disease. They found that genetic or pharmacological inhibition of the ghrelin receptor eliminated the anorectic effect of cabergoline. As ghrelin antagonists normally induce anorexia, this counterintuitive observation suggests that signaling through ghrelin and dopamine D2 receptors was altered by heterodimerization, supporting the functional relevance of the heterodimers.

As deficits in dopamine-mediated neurotransmission are linked to several brain disorders, the existence of these heterodimers could have therapeutic implications. For example, ghrelin antagonists could be used to target brain areas that contain heterodimers while sparing those that only have dopamine D2 receptors. This might result in a more selective neurological outcome or fewer side effects than the use of a dopamine D2 antagonist. $-J C L$

\section{CANCER}

\section{Choked tumors bounce back}

Therapies targeting a tumor's blood supply have been proposed to have an undesirable side effect: promoting metastasis. Mechanistic insights into this controversial caveat are provided by two recent papers.

Cooke et al. (Cancer Cel/ 21, 66-81) examined the contribution of pericytes, the cells that wrap around and support blood vessels, to cancer progression in people with and in mouse models of breast cancer. Although pericyte ablation did not affect primary tumor growth, it increased metastasis in mouse models, and lower pericyte coverage of blood ves-

NEURODEGENERATION

\title{
Prion escape to the spleen
}

Prions are infectious agents that can propagate and accumulate in brain and lymphoid tissues, causing devastating neurodegenerative disorders. Their ability to cross the transmission barrier from one species to another is low; but this barrier can be overcome, leading to diseases in humans, such as variant Creutzfeldt-Jakob disease. Although this cross-species transmission is currently gauged by the presence of clinical signs and of the prion in the brain of the new host, a new study calls for closer scrutiny of foreign prions in extraneuronal tissues (Science, 335, 472-475).

The authors used three mouse models-two strains that transgenically expressed ovine prion protein and one that expressed the human prion protein-to compare the efficacy of cross-species transmission in brain and spleen. Intracerebral inoculation of a foreign prion resulted in no or little infectious agent in the brains of these mice. However, the infectious foreign prions were found in the spleen, even at earlier time points after inoculation than when neurotropic prions were used, indicating that lymphoid tissue may be more permissive for the propagation of foreign prions compared to the brain.

What causes this preferential prion replication in lymphoid tissues is not yet clear; however, the findings indicate that the human species barrier to foreign prions may be leakier than previously estimated on the basis of the presence of prions in brain, and silent carriers of prion disease may therefore have been overlooked. $-C P$

sels correlated with poor prognosis in humans with breast cancer. The authors unraveled a mechanism by which, in the absence of pericytes, tumor blood vessel function is affected, leading to decreased perfusion and hypoxia. Hypoxia-activated signaling pathways involving the oncogenic Met receptor then trigger a phenotypic adaptation that drives tumor cell invasion and metastasis. The authors also report that drugs that target pericytes through inhibition of platelet-derived growth factor receptor may help drive hypoxia-driven metastatic spread, and they suggest that combining these drugs with Met inhibitors may prevent unsought clinical complications.

Other promalignant effects of drug-induced hypoxia are deciphered in a recent report (Proc. Natl. Acad. Sci. USA doi:10.1073/ pnas.1018866109). In mice, Conley et al. show that antiangiogenic drugs targeting blood vessels can also induce hypoxia. In this case, low oxygen concentrations trigger signaling pathways that lead to the expansion of a tumor cell population with stem-like properties. These tumor-initiating cells have previously been shown to have metastatic features and may be responsible for therapeutic resistance and relapse.
Further work is needed to clarify the effects of tumor vessel-targeting drugs, but both reports support the hypothesis that one of their potential effects, hypoxia, can promote adaptations in cancers that result in increased malignancy. Perhaps making life hard for tumors may end up making them harder to kill.-VA

\section{METABOLIC DISORDERS FGF21 action in the fat}

Fibroblast growth factor 21 (FGF21) is a potent hormone with antiobesity and antidiabetic effects, and it has been the focus of clinical trials. But how the protein works, especially in vivo, is still mysterious. Paul A. Dutchak et al. (Cell 148, 556-567) now provide important insights into this mechanism. They find that in response to feeding FGF21 is expressed in white adipose tissue, where it acts locally in fat cells to increase peroxisome proliferatoractivated receptor- $\gamma$ (PPAR- $\gamma$ ) activity. PPAR- $\gamma$ is a key mediator of adipocyte maturation and function, and the team found that FGF21knockout mice have a mild lipodystrophic phenotype. Although they did not report on such 